Korean J. Math. 22 (2014), No. 3, pp. 471-489

http://dx.doi.org/10.11568/kjm.2014.22.3.471

\title{
HAMILTONIAN SYSTEM WITH THE SUPERQUADRATIC NONLINEARITY AND THE LIMIT RELATIVE CATEGORY THEORY
}

\author{
TACKSun JunG* AND Q-Heung ChOI ${ }^{\dagger}$
}

\begin{abstract}
We investigate the number of the weak periodic solutions for the bifurcation problem of the Hamiltonian system with the superquadratic nonlinearity. We get one theorem which shows the existence of at least two weak periodic solutions for this system. We obtain this result by using variational method, critical point theory induced from the limit relative category theory.
\end{abstract}

\section{Introduction}

Let $G(t, z(t))$ be a $C^{2}$ function defined on $R^{1} \times R^{2 n}$ which is $2 \pi$-periodic with respect to the first variable $t$ and $\lambda \in R$. In this paper we investigate the multiplicity of the $2 \pi$-periodic solutions for the bifurcation problem of the following Hamiltonian system

$$
\dot{p(t)}=-\lambda q(t)-G_{q}(t, p(t), q(t))
$$

Received July 11, 2014. Revised September 15, 2014. Accepted September 15, 2014.

2010 Mathematics Subject Classification: 35Q70, 35F50.

Key words and phrases: Hamiltonian system, bifurcation problem, superquadratic nonlinearity, variational method, critical point theory induced from the limit relative category theory, $(P . S .)_{c}^{*}$ condition.

* Corresponding author.

$\dagger$ This work was supported by Basic Science Research Program through the National Research Foundation of Korea(NRF) funded by the Ministry of Education, Science and Technology (KRF-2013010343).

(c) The Kangwon-Kyungki Mathematical Society, 2014.

This is an Open Access article distributed under the terms of the Creative commons Attribution Non-Commercial License (http://creativecommons.org/licenses/by -nc/3.0/) which permits unrestricted non-commercial use, distribution and reproduction in any medium, provided the original work is properly cited. 


$$
\dot{q(t)}=\lambda p(t)+G_{p}(t, p(t), q(t)),
$$

where $p, q \in R^{n}$. Let $z=(p, q)$ and $J$ be the standard symplectic structure on $R^{2 n}$, i. e.,

$$
J=\left(\begin{array}{rr}
0 & -I_{n} \\
I_{n} & 0
\end{array}\right)
$$

where $I_{n}$ is the $n \times n$ identity matrix. Then (1.1) can be rewritten as

$$
-J \dot{z}=\lambda z+G_{z}(t, z(t)),
$$

where $\dot{z}=\frac{d z}{d t}$ and $G_{z}$ is the gradient of $G$. We assume that $G \in C^{2}\left(R^{1} \times\right.$ $\left.R^{2 n}, R^{1}\right)$ satisfies the following conditions:

(G1) $G \in C^{2}\left(R^{1} \times R^{2 n}, R\right), G(0, \theta)=0$, where $\theta=(0, \cdots, 0)$.

(G2) There exist $1<p_{1} \leq p_{2}<2 p_{1}+1, \alpha_{1}>0, \alpha_{2}>0, \beta_{1} \geq 0$ such that

$\alpha_{1}\|z(t)\|_{R^{2 n}}^{p_{1}+1}-\beta_{1} \leq G(t, z(t)) \leq \alpha_{2}\|z(t)\|_{R^{2 n}}^{p_{2}+1}, \quad$ for every $z \in R^{2 n}$.

(G3) $G$ is $2 \pi$-periodic function with respect to $t$.

Several authors ([1], [5], [6], [7] etc.) studied the nonlinear Hamiltonian system. Jung and Choi ([5], [6], [7]) considered (1.1) with nonsingular potential nonlinearity or jumping nonlinearity crossing one eigenvalue, or two eigenvalues, or several eigenvalues. Chang ([1]) proved that (1.1) has at least two nontrivial $2 \pi$-periodic weak solutions under some asymptotic nonlinearity. Jung and Choi ([5]) proved that (1.1) has at least $m$ weak solutions, which are geometrically distinct and nonconstant under some jumping nonlinearity.

We are looking for the weak solutions of (1.1) under the conditions $(G 1)$-(G3). The $2 \pi$-periodic weak solution $z=(p, q) \in E$ of (1.1) satisfies

$$
\int_{0}^{2 \pi}\left(\dot{z}-\lambda z(t)-J G_{z}(t, z(t))\right) \cdot J w d t=0 \quad \text { for all } w \in E
$$

i.e.,

$$
\begin{array}{r}
\int_{0}^{2 \pi}\left[\left(\dot{p}+\lambda q(t)+G_{q}(t, z(t))\right) \cdot \psi-\left(\dot{q}-\lambda p(t)-G_{p}(t, z(t))\right) \cdot \phi\right] d t=0 \\
\text { for all } \zeta=(\phi, \psi) \in E
\end{array}
$$

where $E$ is introduced in section 2 .

Our main result is as follows: 
Theorem 1.1. Assume that $G$ satisfies the conditions $(G 1)-(G 3)$, and that $j_{0}, j_{1}$ are negative integers with $j_{1}<j_{0}<0$. Then there exists a small number $\delta>0$ such that for any $\lambda$ with $j_{1}-\delta<\lambda<j_{1}<j_{0}<0$, system (1.1) has at least two nontrivial $2 \pi$-periodic solutions.

The outline of the proof of Theorem 1.1 is as follows: In section 2, we introduce the perturbed operator $A_{\epsilon}=\epsilon I+A$ of the operator $A(A(z(t))=-J \dot{z}(t))$ because of the lack of the compactness of the operator $A^{-1}$, approach the variational method, obtain some results on the corresponding functional of the perturbed problem $A_{\epsilon}(z)=\lambda z+\epsilon z+$ $G_{z}(t, z(t))$ of (1.1) and recall the critical point theory induced from the limit relative category, which plays a crucial role to prove the multiplicity result. In section 3, we prove Theorem 1.1 by the critical point theory induced from the limit relative category.

\section{Variational Formulation}

Let $L^{2}\left([0,2 \pi], R^{2 n}\right)$ denote the set of $2 n$-tuples of the square integrable $2 \pi$-periodic functions and choose $z \in L^{2}\left([0,2 \pi], R^{2 n}\right)$. Then it has a Fourier expansion $z(t)=\sum_{k=-\infty}^{k=+\infty} a_{k} e^{i k t}$, with $a_{k}=\frac{1}{2 \pi} \int_{0}^{2 \pi} z(t) e^{-i k t} d t \in$ $C^{2 n}, a_{-k}=\overline{a_{k}}$ and $\sum_{k \in Z}\left|a_{k}\right|^{2}<\infty$. Let

$$
A: z(t) \mapsto-J \dot{z}(t)
$$

with domain

$$
\begin{aligned}
D(A) & =\left\{z(t) \in H^{1}\left([0,2 \pi], R^{2 n}\right) \mid z(0)=z(2 \pi)\right\} \\
& =\left\{\left.z(t) \in L^{2}\left([0,2 \pi], R^{2 n}\right)\left|\sum_{k \in Z}(\epsilon+|k|)^{2}\right| a_{k}\right|^{2}<+\infty\right\},
\end{aligned}
$$

where $\epsilon$ is a positive small number. Then $A$ is self-adjoint operator. Let $\left\{M_{\lambda}\right\}$ be the spectral resolution of $A$, and let $\tau$ be a positive number such that $\tau \notin \sigma(A)$ and $[-\tau, \tau]$ contains only one element 0 of $\sigma(A)$. Let

$$
\begin{gathered}
P_{0}=\int_{-\tau}^{\tau} d M_{\lambda}, \quad P_{+}=\int_{\tau}^{+\infty} d M_{\lambda}, \quad P_{-}=\int_{-\infty}^{-\tau} d M_{\lambda}, \\
P_{\left(-\infty, j_{1}-1\right]}=\int_{-\infty}^{j_{1}-1} d M_{\lambda}, \quad P_{\left[j_{1}, j_{0}\right]}=\int_{j_{1}}^{j_{0}} d M_{\lambda} .
\end{gathered}
$$

Let

$L_{0}=P_{0} L^{2}\left([0,2 \pi], R^{2 n}\right), L_{+}=P_{+} L^{2}\left([0,2 \pi], R^{2 n}\right), L_{-}=P_{-} L^{2}\left([0,2 \pi], R^{2 n}\right)$, 
$L_{\left(-\infty, j_{1}-1\right]}=P_{\left(-\infty, j_{1}-1\right]} L^{2}\left([0,2 \pi], R^{2 n}\right), \quad L_{\left[j_{1}, j_{0}\right]}=P_{\left[j_{1}, j_{0}\right]} L^{2}\left([0,2 \pi], R^{2 n}\right)$.

For each $u \in L^{2}\left([0,2 \pi], R^{2 n}\right)$, we have the composition

$$
u=u_{0}+u_{+}+u_{-},
$$

where $u_{0} \in L_{0}, u_{+} \in L_{+}, u_{-} \in L_{-}$. According to $A$, there exists a small number $\epsilon>0$ such that $-\epsilon \notin \sigma(A)$. Let us define the space $E$ as follows:

$$
E=D\left(|A|^{\frac{1}{2}}\right)=\left\{\left.z \in L^{2}\left([0,2 \pi], R^{2 n}\right)\left|\sum_{k \in Z}(\epsilon+|k|)\right| a_{k}\right|^{2}<\infty\right\}
$$

with the scalar product

$$
(z, w)_{E}=\epsilon(z, w)_{L^{2}}+\left(|A|^{\frac{1}{2}} z,|A|^{\frac{1}{2}} w\right)_{L^{2}}
$$

and the norm

$$
\|z\|=(z, z)_{E}^{\frac{1}{2}}=\left(\sum_{k \in Z}(\epsilon+|k|)\left|a_{k}\right|^{2}\right)^{\frac{1}{2}} .
$$

The space $E$ endowed with this norm is a real Hilbert space continuously embedded in $L^{2}\left([0,2 \pi], R^{2 n}\right)$. The scalar product in $L^{2}$ naturally extends as the duality pairing between $E$ and $E^{\prime}=W^{-\frac{1}{2}, 2}\left([0,2 \pi], R^{2 n}\right)$. We note that the operator $(\epsilon+|A|)^{-1}$ is a compact linear operator from $L^{2}\left([0,2 \pi], R^{2 n}\right)$ to $E$ such that

$$
\left((\epsilon+|A|)^{-1} w, z\right)_{E}=\int_{0}^{2 \pi}(w(t), z(t)) d t .
$$

Let

$$
A_{\epsilon}=\epsilon I+A \text {. }
$$

Let

$$
\begin{gathered}
E_{0}=\left|A_{\epsilon}\right|^{-\frac{1}{2}} L_{0}, \quad E_{+}=\left|A_{\epsilon}\right|^{-\frac{1}{2}} L_{+}, \quad E_{-}=\left|A_{\epsilon}\right|^{-\frac{1}{2}} L_{-}, \\
E_{\left(-\infty, j_{1}-1\right]}=\left|A_{\epsilon}\right|^{-\frac{1}{2}} L_{\left(-\infty, j_{1}-1\right]}, \quad E_{\left[j_{1}, j_{0}\right]}=\left|A_{\epsilon}\right|^{-\frac{1}{2}} L_{\left[j_{1}, j_{0}\right]} .
\end{gathered}
$$

Then $E=E_{0} \oplus E_{+} \oplus E_{-}$, and for $z \in E$, $z$ has the decomposition $z=z_{0}+z_{+}+z_{-} \in E$, where

$$
z_{0}=\left|A_{\epsilon}\right|^{-\frac{1}{2}} u_{0}, \quad z_{+}=\left|A_{\epsilon}\right|^{-\frac{1}{2}} u_{+}, \quad z_{-}=\left|A_{\epsilon}\right|^{-\frac{1}{2}} u_{-} .
$$

Thus we have

$$
\left\|z_{0}\right\|_{E_{0}}=\left\|u_{0}\right\|_{L_{0}}, \quad\left\|z_{+}\right\|_{E_{+}}=\left\|u_{+}\right\|_{L_{+}}, \quad\left\|z_{-}\right\|_{E_{-}}=\left\|u_{-}\right\|_{L_{-}}
$$


and that $E_{0}, E_{+}, E_{-}$are isomorphic to $L_{0}, L_{+}, L_{-}$, respectively. Moreover $E=E_{\left(-\infty, j_{1}-1\right]} \oplus E_{\left[j_{1}, j_{2}\right]} \oplus E_{+}$. Let us define a functional

$$
f(u)=\frac{1}{2}\left(\left\|u_{+}\right\|_{L^{2}}^{2}+\left\|M_{+} u_{0}\right\|_{L^{2}}^{2}-\left\|M_{-} u_{0}\right\|_{L^{2}}^{2}-\left\|u_{-}\right\|_{L^{2}}^{2}\right)-\psi_{\epsilon}(z),
$$

where $\psi_{\epsilon}(z)=\psi(z)+\frac{\epsilon}{2}\|z\|_{L^{2}}^{2}, \psi(z)=\int_{0}^{2 \pi}\left[\frac{\lambda}{2} z(t)^{2}+G(t, z(t))\right] d t, M_{+}=$ $\int_{0}^{\infty} d M_{\lambda}, M_{-}=\int_{-\infty}^{0} d M_{\lambda}$. By $G \in C^{2}, \psi(z)=\int_{0}^{2 \pi}\left[\frac{\lambda}{2} z(t)^{2}+G(t, z(t))\right] \in$ $C^{2}$. Let

$F(z)=\lambda z(t)+G_{z}(t, z(t)), \quad F_{\epsilon}(z)=\epsilon z+F(z)=\epsilon z+\lambda z(t)+G_{z}(t, z(t))$.

Then (1.2) can be rewritten as

$$
A_{\epsilon}(z)=F_{\epsilon}(z) \text {. }
$$

The Euler equation of the functional $f(u)$ is the system

$$
\begin{gathered}
u_{+}=\left|A_{\epsilon}\right|^{-\frac{1}{2}} P_{+} F_{\epsilon}(z), \\
u_{-}=-\left|A_{\epsilon}\right|^{-\frac{1}{2}} P_{-} F_{\epsilon}(z), \\
M_{+} u_{0}=\left|A_{\epsilon}\right|^{-\frac{1}{2}} M_{+} P_{0} F_{\epsilon}(z) \quad M_{-} u_{0}=-\left|A_{\epsilon}\right|^{-\frac{1}{2}} M_{-} P_{0} F_{\epsilon}(z) .
\end{gathered}
$$

The system (2.3)-(2.5) is reduced to

$$
\begin{aligned}
& A_{\epsilon} z_{+}=P_{+} F_{\epsilon}\left(z_{0}+z_{+}+z_{-}\right) \quad \text { or } \quad z_{+}=\left(A_{\epsilon}\right)^{-1} P_{+} F_{\epsilon}\left(z_{0}+z_{+}+z_{-}\right), \quad(2.6) \\
& A_{\epsilon} z_{-}=P_{-} F_{\epsilon}\left(z_{0}+z_{+}+z_{-}\right) \quad \text { or } \quad z_{-}=\left(A_{\epsilon}\right)^{-1} P_{-} F_{\epsilon}\left(z_{0}+z_{+}+z_{-}\right),(2.7) \\
& A_{\epsilon} M_{+} z_{0}=M_{+} P_{0} F_{\epsilon}\left(z_{0}+z_{+}+z_{-}\right), A_{\epsilon} M_{-} z_{0}=M_{-} P_{0} F_{\epsilon}\left(z_{0}+z_{+}+z_{-}\right),
\end{aligned}
$$

It follows from (2.3)-(2.8) that $z=z_{0}+z_{+}+z_{-}$is a solution of (1.2) if and only if $u=u_{0}+u_{+}+u_{-}$is a critical point of $f$. By (2.1), we define a functional

$$
I(z)=f(u(z)) .
$$

The functional $I(z)$ is of the form

$$
\begin{array}{r}
I(z)=\frac{1}{2}\left(\left\|\left|A_{\epsilon}\right|^{\frac{1}{2}} z_{+}\right\|_{L^{2}}^{2}+\left\|\left|A_{\epsilon}\right|^{\frac{1}{2}} M_{+} z_{0}\right\|_{L^{2}}^{2}-\left\|\left(-\left|A_{\epsilon}\right|\right)^{\frac{1}{2}} z_{-}\right\|_{L^{2}}^{2}\right. \\
\left.-\left\|\left(-\left|A_{\epsilon}\right|\right)^{\frac{1}{2}} M_{-} z_{0}\right\|_{L^{2}}^{2}\right)-\psi_{\epsilon}(z) .
\end{array}
$$

Thus it suffices to find the critical points of the functional $I$ to find the critical points of the functional $f$. By the following Lemma 2.1, the weak solutions of (2.2) coincide with the critical points of the functional $I(z)$. 
Lemma 2.1. Assume that $G$ satisfies the conditions $(G 1)-(G 3)$ and $\lambda \notin \sigma(A), \lambda<0$. Then $I(z)$ is continuous and Fréchet differentiable in $E$ with Fréchet derivative

$D I(z) w=\int_{0}^{2 \pi}\left(J A_{\epsilon} z(t)-(\lambda+\epsilon) J z(t)-J G_{z}(t, z(t))\right) \cdot J w d t$ for all $w \in E$,

Moreover $D I \in C$. That is, $I \in C^{1}$.

Proof. First we prove that $I(z)=\int_{0}^{2 \pi}\left[\frac{1}{2} A_{\epsilon} z-\frac{\epsilon+\lambda}{2} z^{2}-G(t, z(t))\right] d t$ is continuous in $E$. For $z, w \in E$,

$$
\begin{aligned}
& |I(z+w)-I(z)| \\
= & \mid \int_{0}^{2 \pi} \frac{1}{2} A_{\epsilon}(z+w) \cdot(z+w)-\int_{0}^{2 \pi}\left[G(t, z+w)+\frac{\epsilon+\lambda}{2}(z+w)^{2}\right] \\
& -\int_{0}^{2 \pi} \frac{1}{2} A_{\epsilon}(z) \cdot z+\int_{0}^{2 \pi}\left[G(t, z)+\frac{\epsilon+\lambda}{2} z^{2}\right] \\
= & \mid \int_{0}^{2 \pi} \frac{1}{2}\left[A_{\epsilon}(z) \cdot w+A_{\epsilon}(w) \cdot z+A_{\epsilon}(w) \cdot w\right] \\
& \left.-\int_{0}^{2 \pi}\left[G(t, z+w)-G(t, z)+\frac{\epsilon+\lambda}{2}\left(2 z \cdot w+w^{2}\right)\right)\right] \mid .
\end{aligned}
$$

We note that

$$
\mid \int_{0}^{2 \pi} \frac{1}{2}\left[A_{\epsilon}(z) \cdot w+A_{\epsilon}(w) \cdot z+A_{\epsilon}(w) \cdot w \mid=O\left(\|w\|_{R^{2 n}}\right)\right.
$$

and

$$
\begin{aligned}
\left|\int_{0}^{2 \pi}[G(t, z+w)-G(t, z)] d t\right| & \leq\left|\int_{0}^{2 \pi}\left[G_{z}(t, z(t)) \cdot w+O\left(\|w\|_{R^{2 n}}\right)\right] d t\right| \\
& =O\left(\|w\|_{R^{2 n}}\right) .
\end{aligned}
$$

Thus we have

$$
|I(z+w)-I(z)|=O\left(\|w\|_{R^{2 n}}\right) .
$$


Next we shall prove that $I(z)$ is Fréchet differentiable in E. For $z, w \in$ $E$,

$$
\begin{aligned}
& |I(z+w)-I(z)-D I(z) w| \\
= & \mid \int_{0}^{2 \pi} \frac{1}{2} A_{\epsilon}(z+w) \cdot(z+w)-\int_{0}^{2 \pi}\left[G(t, z+w)+\frac{\epsilon+\lambda}{2}(z+w)^{2}\right] \\
& -\int_{0}^{2 \pi} \frac{1}{2} A_{\epsilon}(z) \cdot z+\int_{0}^{2 \pi}\left[G(t, z)+\frac{\epsilon+\lambda}{2} z^{2}\right] \\
& -\int_{0}^{2 \pi} J A_{\epsilon}(z) \cdot J w+\int_{0}^{2 \pi}\left[J G_{z}(t, z) \cdot J w+(\epsilon+\lambda) J z \cdot J w\right] \mid \\
= & \mid \int_{0}^{2 \pi} \frac{1}{2}\left[A_{\epsilon}(w) \cdot z+A_{\epsilon}(w) \cdot w\right] \\
& \left.-\int_{0}^{2 \pi}\left[G(t, z+w)-G(t, z)-G_{z}(t, z) \cdot w+\frac{\epsilon+\lambda}{2} w^{2}\right)\right] \mid .
\end{aligned}
$$

By (2.10), we have

$$
\int_{0}^{2 \pi}\left[G(t, z+w)-G(t, z)-G_{z}(t, z)\right] d t=O\left(\|w\|_{R^{2 n}}\right) .
$$

Thus

$$
|I(z+w)-I(z)-D I(z) w|=O\left(\|w\|_{R^{2 n}}\right)
$$

Now, we recall the critical point theory on the manifold with boundary. Let $E$ be a Hilbert space and $M$ be the closure of an open subset of $E$ such that $M$ can be endowed with the structure of $C^{2}$ manifold with boundary. Let $f: W \rightarrow R$ be a $C^{1,1}$ functional, where $W$ is an open set containing $M$. For applying the usual topological methods of the critical points theory we need a suitable notion of critical point for $f$ on $M$. We recall the following notions: lower gradient of $f$ on $M,(P . S .)_{c}^{*}$ condition and the limit relative category (see [4]).

Definition 2.1. If $z \in M$, the lower gradient of $f$ on $M$ at $z$ is defined by

$\operatorname{grad}_{M}^{-} f(z)= \begin{cases}D f(z) & \text { if } z \in \operatorname{int}(M), \\ D f(z)+[<D f(z), \nu(z)>]^{-} \nu(z) & \text { if } z \in \partial M\end{cases}$

where we denote by $\nu(z)$ the unit normal vector to $\partial M$ at the point $z$, pointing outwards. We say that $z$ is a lower critical for $f$ on $M$, if 
$\operatorname{grad}_{M}^{-} f(z)=0$.

Since the functional $I(z)$ is strongly indefinite, the notion of the $(P . S .)_{c}^{*}$ condition and the limit relative category is a very useful tool for the proof of the main theorems.

Let $\left(E_{n}\right)_{n}$ be a sequence of closed subspaces of $E$ with the conditions:

$$
E_{n}=E_{n_{-}} \oplus E_{0} \oplus E_{n+} \text {, where } E_{n+} \subset E_{+}, E_{n_{-}} \subset E_{-} \text {for all } n,
$$

$$
\left(E_{n_{+}} \text {and } E_{n_{-}} \text {are subspaces of } E\right) \text {, }
$$

$$
\operatorname{dim} E_{n}<+\infty, E_{n} \subset E_{n+1}, \cup_{n \in N} E_{n} \text { is dense in } E .
$$

Let $P_{E_{n}}$ be the orthogonal projections from $E$ onto $E_{n} . M_{n}=M \cap E_{n}$, for any $n$, be the closure of an open subset of $E_{n}$ and has the structure of a $C^{2}$ manifold with boundary in $E_{n}$. We assume that for any $n$ there exists a retraction $r_{n}: M \rightarrow M_{n}$. For given $B \subset E$, we will write $B_{n}=B \cap E_{n}$.

Definition 2.2. Let $c \in R$. We say that $f$ satisfies the $(P . S .)_{c}^{*}$ condition with respect to $\left(M_{n}\right)_{n}$, on the manifold $M$ with boundary, if for any sequence $\left(k_{n}\right)_{n}$ in $N$ and any sequence $\left(u_{n}\right)_{n}$ in $M$ such that $k_{n} \rightarrow \infty, u_{n} \in M_{k_{n}}, \forall n, f\left(u_{n}\right) \rightarrow c, \operatorname{grad}_{M_{k_{n}}}^{-} f\left(u_{n}\right) \rightarrow 0$, there exists a subsequence of $\left(u_{n}\right)_{n}$ which converges to a point $u \in M$ such that $\operatorname{grad}_{M}^{-} f(u)=0$.

Let $Y$ be a closed subspace of $M$.

Definition 2.3. $\quad$ Let $B$ be a closed subset of $M$ with $Y \subset B$. We define the relative category $\operatorname{cat}_{M, Y}(B)$ of $B$ in $(\mathrm{M}, \mathrm{Y})$, as the least integer $h$ such that there exist $h+1$ closed subsets $U_{0}, U_{1}, \ldots, U_{h}$ with the following properties:

$B \subset U_{0} \cup U_{1} \cup \ldots \cup U_{h}$;

$U_{1}, \ldots, U_{h}$ are contractible in $M$;

$Y \subset U_{0}$ and there exists a continuous map $F: U_{0} \times[0,1] \rightarrow M$ such that

$$
\begin{aligned}
F(x, 0)=x & \forall x \in U_{0}, \\
F(x, t) \in Y & \forall x \in Y, \forall t \in[0,1], \\
F(x, 1) \in Y & \forall x \in U_{0} .
\end{aligned}
$$

If such an $h$ does not exist, we say that $\operatorname{cat}_{M, Y}(B)=+\infty$. 
Definition 2.4. Let $(X, Y)$ be a topological pair and $\left(X_{n}\right)_{n}$ be a sequence of subsets of $X$. For any subset $B$ of $X$ we define the limit relative category of $B$ in $(X, Y)$, with respect to $\left(X_{n}\right)_{n}$, by

$$
\operatorname{cat}_{(X, Y)}^{*}(B)=\lim \sup _{n \rightarrow \infty} \operatorname{cat}_{\left(X_{n}, Y_{n}\right)}\left(B_{n}\right) \text {. }
$$

Now we consider a theorem which gives an estimate of the number of critical points of a functional, in terms of the limit relative category of its sublevels. The theorem is proved repeating the classical arguments, using the nonsmooth version of the classical Deformation Lemma for functions on manifolds with boundary.

Let $Y$ be a fixed subset of $M$. We set

$$
\begin{gathered}
\mathcal{B}_{\mathrm{i}}=\left\{\mathrm{B} \subset \mathrm{M} \mid \operatorname{cat}_{(\mathrm{M}, \mathrm{Y})}^{*}(\mathrm{~B}) \geq \mathrm{i}\right\}, \\
c_{i}=\inf _{B \in \mathcal{B}_{\mathrm{i}}} \sup _{x \in B} f(x) .
\end{gathered}
$$

We have the following multiplicity theorem.

TheOREM 2.1. Let $i \in N$ and assume that

(1) $c_{i}<+\infty$,

(2) $\sup _{x \in Y} f(x)<c_{i}$,

(3) the (P.S. $)_{c_{i}}^{*}$ condition with respect to $\left(M_{n}\right)_{n}$ holds.

Then there exists a lower critical point $x$ such that $f(x)=c_{i}$. If

$$
c_{i}=c_{i+1}=\ldots=c_{i+k-1}=c,
$$

then

$$
\operatorname{cat}_{M}\left(\left\{x \in M \mid f(x)=c, \operatorname{grad}_{M}^{-} f(x)=0\right\}\right) \geq k .
$$

Proof. Let $c=c_{i}$; using the $(P . S .)_{c}^{*}$ condition, with respect to $\left(M_{n}\right)_{n}$, one can prove that, for any neighborhood $N$ of

$$
K_{c}=\left\{x \mid f(x)=c, \operatorname{grad}_{M}^{-} f(x)=0\right\},
$$

there exist $n_{0}$ in $N$ and $\delta>0$ such that $\left\|\operatorname{grad}_{M}^{-}\right\| \geq \delta$ for all $n \geq n_{0}$ and all $x \in E_{n} \backslash N$ with $c-\delta \leq f(x) \leq c+\delta$. Moreover it is not difficult to see that, for all $n$, the function $\tilde{f}_{n}: E_{n} \rightarrow R \cup\{+\infty\}$ defined by $\tilde{f}_{n}=f(x)$, if $x \in M_{n}, \tilde{f}_{n}(x)=+\infty$, otherwise, is $\phi$-convex of order two, according to the definitions of [7]. Then the conclusion follows using the same arguments of $[1,8]$ and the nonsmooth version of the classical Deformation Lemma. 
Now we state the following multiplicity result (for the proof see Theorem 4.6 of [8]) which will be used in the proof of our main theorem.

Theorem 2.2. Let $E$ be a Hilbert space and let $E=X_{1} \oplus X_{2} \oplus X_{3}$, where $X_{1}, X_{2}, X_{3}$ are three closed subspaces of $E$ with $X_{1}, X_{2}$ of finite dimension. For a given subspace $X$ of $E$, let $P_{X}$ be the orthogonal projection from $E$ onto $X$. Set

$$
C=\left\{x \in E \mid\left\|P_{X_{2}} x\right\| \geq 1\right\}
$$

and let $f: W \rightarrow R$ be a $C^{1,1}$ function defined on a neighborhood $W$ of $C$. Let $1<\rho<R, R_{1}>0$. We define

$$
\begin{aligned}
\Delta= & \left\{x_{1}+x_{2} \mid x_{1} \in X_{1}, x_{2} \in X_{2},\left\|x_{1}\right\| \leq R_{1}, 1 \leq\left\|x_{2}\right\| \leq R\right\}, \\
\Sigma= & \left\{x_{1}+x_{2} \mid x_{1} \in X_{1}, x_{2} \in X_{2},\left\|x_{1}\right\| \leq R_{1},\left\|x_{2}\right\|=1\right\} \\
& \cup\left\{x_{1}+x_{2} \mid x_{1} \in X_{1}, x_{2} \in X_{2},\left\|x_{1}\right\| \leq R_{1},\left\|x_{2}\right\|=R\right\} \\
& \cup\left\{x_{1}+x_{2} \mid x_{1} \in X_{1}, x_{2} \in X_{2},\left\|x_{1}\right\|=R_{1}, 1 \leq\left\|x_{2}\right\| \leq R\right\}, \\
S= & \left\{x \in X_{2} \oplus X_{3} \mid\|x\|=\rho\right\}, \\
B= & \left\{x \in X_{2} \oplus X_{3} \mid\|x\| \leq \rho\right\} .
\end{aligned}
$$

(a) Assume that

$$
\sup f(\Sigma)<\inf f(S)
$$

and (b) that the (P.S. $)_{c}$ condition holds for $f$ on $C$, with respect to the sequence $\left(C_{n}\right)_{n}, \forall c \in[\alpha, \beta]$, where

$$
\alpha=\inf f(S), \quad \beta=\sup f(\Delta) .
$$

(c) Moreover we assume $\beta<+\infty$ and $\left.f\right|_{X_{1} \oplus X_{3}}$ has no critical points $z$ in $X_{1} \oplus X_{3}$ with $\alpha \leq f(z) \leq \beta$.

Then there exist two lower critical points $z_{1}, z_{2}$ for $f$ on $C$ such that $\alpha \leq f\left(z_{i}\right) \leq \beta, i=1.2$.

\section{Proof of Theorem 1.1}

Throughout this section we assume that $G$ satisfies the conditions $(G 1)-(G 3), \lambda \notin Z$ and $\lambda<0$. Let $I(z)$ be the functional defined in section 2, i.e.,

$$
\begin{array}{r}
I(z)=\frac{1}{2}\left(\left\|\left|A_{\epsilon}\right|^{\frac{1}{2}} z_{+}\right\|_{L^{2}}^{2}+\left\|\left|A_{\epsilon}\right|^{\frac{1}{2}} M_{+} z_{0}\right\|_{L^{2}}^{2}-\left\|\left(-\left|A_{\epsilon}\right|\right)^{\frac{1}{2}} z_{-}\right\|_{L^{2}}^{2}\right. \\
\left.-\left\|\left(-\left|A_{\epsilon}\right|\right)^{\frac{1}{2}} M_{-} z_{0}\right\|_{L^{2}}^{2}\right)-\psi_{\epsilon}(z),
\end{array}
$$


where $\psi_{\epsilon}(z)=\psi(z)+\frac{\epsilon}{2}\|z\|_{L^{2}}^{2}, \psi(z)=\int_{0}^{2 \pi}\left[\frac{\lambda}{2} z(t)^{2}+G(t, z(t))\right] d t, M_{+}=$ $\int_{0}^{\infty} d M_{\lambda}, M_{-}=\int_{-\infty}^{0} d M_{\lambda}$.

We shall show that the functional $I(z)$ satisfies the geometric assumptions of Theorem 2.2.

Lemma 3.1. ((P.S. $)^{*}$ condition)

Assume that $G$ satisfies the conditions $(G 1)-(G 3)$ and $\lambda \notin Z$. Then $I(z)$ satisfies the $(P . S .)_{\gamma}^{*}$ condition with respect to $\left(E_{n}\right)_{n}$, for any $\gamma \in R$.

Proof. Let $\left(k_{n}\right)_{n}$ and $\left(z_{n}\right)_{n}$ be two sequences such that $k_{n} \rightarrow+\infty$, and for any sequence $\left(z_{n}\right)_{n}$ with $z_{n} \in E_{k_{n}}$,

$$
I\left(z_{n}\right) \rightarrow \gamma
$$

and

$$
D I_{k_{n}}\left(z_{n}\right) \rightarrow \theta
$$

where $I_{k_{n}}$ is a restriction of $I$ on $E_{k_{n}}$ and $\theta=(0, \cdots, 0)$. It follows from $D I_{k_{n}}\left(z_{n}\right) \rightarrow 0$ that

$$
P_{E_{k_{n}}} z_{n}-P_{E_{k_{n}}} A_{\epsilon}^{-1}\left((\lambda+\epsilon) z_{n}+G_{z}\left(t, z_{n}(t)\right)\right) \longrightarrow \theta
$$

where $A_{\epsilon}^{-1}$ is a compact operator. We claim that $\left(z_{n}\right)_{n}$ is bounded. By contradiction, we suppose that $\left\|z_{n}\right\| \rightarrow \infty$. If $w_{n}=\frac{z_{n}}{\left\|z_{n}\right\|}$, we can suppose that $w_{n} \rightarrow w_{0}$ weakly for some $w_{0} \in E$. We have

$$
\begin{gathered}
0 \longleftarrow\left\langle P_{E_{k_{n}}} w_{n}-A_{\epsilon}^{-1} P_{E_{k_{n}}}\left((\lambda+\epsilon) w_{n}+\frac{G_{z}\left(t, z_{n}(t)\right)}{\left\|z_{n}\right\|}\right), w_{n}\right\rangle \\
=P_{E_{k_{n}}}\left\langle w_{n}, w_{n}\right\rangle-\left\langle A_{\epsilon}^{-1} P_{E_{k_{n}}}\left((\lambda+\epsilon) w_{n}+\frac{G_{z}\left(t, z_{n}(t)\right)}{\left\|z_{n}\right\|}\right), w_{n}\right\rangle .
\end{gathered}
$$

Since $A_{\epsilon}^{-1}$ is a compact operator, $(\lambda+\epsilon) w_{n}$ is bounded and $\frac{G_{z}\left(t, z_{n}(t)\right)}{\left\|z_{n}\right\|} \rightarrow 0$, $A_{\epsilon}^{-1}\left(P_{E_{k_{n}}}\left((\lambda+\epsilon) w_{n}+\frac{G_{z}\left(t, z_{n}(t)\right)}{\left\|z_{n}\right\|}\right)\right)$ converges to $A_{\epsilon}^{-1}\left((\lambda+\epsilon) w_{0}\right)$ and we have

$$
\left.0=\left\langle w_{0}, w_{0}\right\rangle-\left\langle A_{\epsilon}^{-1}\left((\lambda+\epsilon) w_{0}\right), w_{0}\right\rangle=\left\|w_{0}\right\|^{2}-\left\langle A_{\epsilon}^{-1}(\lambda+\epsilon)\right) w_{0}, w_{0}\right\rangle,
$$

from which $w_{0}$ is a solution of the equation

$$
A_{\epsilon} w=(\lambda+\epsilon) w .
$$

Since $\lambda \notin \sigma(A), w_{0}=0$, which is a contradiction to the fact that $\left\|w_{0}\right\|=$ 1. Thus $\left(z_{n}\right)_{n}$ is bounded. We can suppose that $z_{n} \rightarrow z_{0}$ weakly in $E$, 
for some $z_{0}$ in $E$. We have

$$
\begin{aligned}
& \left\langle P_{E_{k_{n}}} D I\left(z_{n}\right), P_{+} z_{n}+P_{-} z_{n}\right\rangle=\left\|P_{E_{k_{n}}} P_{+} z_{n}\right\|^{2}-\left\|P_{E_{k_{n}}} P_{-} z_{n}\right\|^{2} \\
& \quad-P_{E_{k_{n}}} \int_{0}^{2 \pi}\left(\lambda z_{n}(t)+\epsilon z_{n}(t)+G_{z}\left(t, z_{n}\right)\right)\left(P_{+} z_{n}+P_{-} z_{n}\right) \rightarrow 0 .
\end{aligned}
$$

By $(G 1)$ and the boundedness of $G_{z}\left(t, z_{n}\right) \cdot\left(P_{+} z_{n}+P_{-} z_{n}\right)$,

$$
\lim _{n \rightarrow \infty}\left\|P_{E_{k_{n}}} P_{+} z_{n}\right\|^{2}-\left\|P_{E_{k_{n}}} P_{-} z_{n}\right\|^{2}=\int_{0}^{2 \pi}\left(\lambda z(t)+\epsilon z(t)+G_{z}(t, z)\right) z,
$$

i.e., $\left\|P_{E_{k_{n}}} P_{+} z_{n}\right\|^{2}-\left\|P_{E_{k_{n}}} P_{-} z_{n}\right\|^{2}$ converges strongly, which implies that, up to a subsequence, $P_{E_{k_{n}}} z_{n}$ converges strongly to $z$, and we prove the lemma and have

$$
D I(z)=\lim _{n \rightarrow \infty} P_{E_{k_{n}}} D I\left(z_{n}\right)=0,
$$

so $z$ is the critical point of $I$.

Let us set

$$
X_{1}=E_{\left(-\infty, j_{1}-1\right]}, \quad X_{2}=E_{\left[j_{1}, j_{0}\right]}, \quad X_{3}=E_{+} .
$$

Then $E$ is the topological direct sum of the subspaces $X_{1}, X_{2}$ and $X_{3}$. Let $P_{X}$ be the orthogonal projection from $E$ onto $X$. Let us set

$$
C=\left\{z \in E \mid\left\|P_{X_{2}} z\right\| \geq 1\right\}
$$

Then $C$ is the smooth manifold with boundary. Let $C_{n}=C \cap E_{n}$. Let us define a functional $\Psi: E \backslash\left\{X_{1} \oplus X_{3}\right\} \rightarrow E$ by

$$
\Psi(z)=z-\frac{P_{X_{2}} z}{\left\|P_{X_{2}} z\right\|}=P_{X_{1} \oplus X_{3}} z+\left(1-\frac{1}{\left\|P_{X_{2}} z\right\|}\right) P_{X_{2}} z .
$$

We have

$$
\nabla \Psi(z) \cdot w=w-\frac{1}{\left\|P_{X_{2}} z\right\|}\left(P_{X_{2}} w-\left\langle\frac{P_{X_{2}} z}{\left\|P_{X_{2}} z\right\|}, w\right\rangle \frac{P_{X_{2}} z}{\left\|P_{X_{2}} z\right\|}\right) .
$$

Let us define the constrained functional $\tilde{I}: C \rightarrow R$ by

$$
\tilde{I}=I \circ \Psi \text {. }
$$

Then $\tilde{I} \in C_{l o c}^{1,1}$. It turns out that

$$
\operatorname{grad}_{C}^{-} \tilde{I}(\tilde{z})=\left\{\begin{array}{l}
P_{X_{1} \oplus X_{3}} D I(z)+\left(1-\frac{1}{\left\|P_{X_{2}} \tilde{z}\right\|_{E}}\right) P_{X_{2}} D I(z) \quad \text { if } z \in \operatorname{int}(C), \\
P_{X_{1} \oplus X_{3}} D I(z)-\left\langle D I(z), \frac{P_{X_{2}} \tilde{z}}{\left\|P_{X_{2}} \tilde{z}\right\|_{E}}\right\rangle^{+} \frac{P_{X_{2}} \tilde{z}}{\left\|P_{X_{2}} \tilde{z}\right\|_{E}} \quad \text { if } z \in \partial C .
\end{array}\right.
$$


We note that if $\tilde{z}$ is the critical point of $\tilde{I}$ and lies in the interior of $C$, then $z=\Psi(\tilde{z})$ is the critical point of $I$. Thus it suffices to find the critical points, which lies in the interior of $C$, for $\tilde{I}$. We also note that

$$
\left\|\operatorname{grad}_{C}^{-} \tilde{I}(\tilde{z})\right\|_{E} \geq\left\|P_{X_{1} \oplus X_{3}} D I(\Psi(\tilde{z}))\right\|_{E} \quad \forall \tilde{z} \in \partial C .
$$

Let us set

$$
\begin{aligned}
S_{23}(\rho)= & \left\{z \in X_{2} \oplus X_{3} \mid\|z\|_{E}=\rho\right\}, \rho>0, \\
S_{23}(\rho)= & \Psi^{-1}\left(S_{23}(\rho)\right), \\
\Delta_{12}\left(R, R_{1}\right)= & \left\{z_{1}+z_{2} \mid z_{1} \in X_{1}, z_{2} \in X_{2},\left\|z_{1}\right\|_{E} \leq R_{1}, 1 \leq\left\|z_{2}\right\|_{E} \leq R\right\}, \\
\Delta_{12}\left(\tilde{R}, R_{1}\right)= & \Psi^{-1}\left(\Delta_{12}\left(R, R_{1}\right)\right) \\
\Sigma_{12}\left(R, R_{1}\right)= & \left\{z_{1}+z_{2} \mid z_{1} \in X_{1}, z_{2} \in X_{2},\left\|z_{1}\right\|_{E} \leq R_{1},\left\|z_{2}\right\|_{E}=1\right\} \\
& \cup\left\{z_{1}+z_{2} \mid z_{1} \in X_{1}, z_{2} \in X_{2},\left\|z_{1}\right\|_{E} \leq R_{1},\left\|z_{2}\right\|_{E}=R\right\} \\
& \cup\left\{z_{1}+z_{2} \mid z_{1} \in X_{1}, z_{2} \in X_{2},\left\|z_{1}\right\|_{E}=R_{1}, 1 \leq\left\|z_{2}\right\|_{E} \leq R\right\}, \\
\Sigma_{12}\left(\tilde{R}, R_{1}\right)= & \Psi^{-1}\left(\Sigma_{12}\left(R, R_{1}\right)\right) .
\end{aligned}
$$

We will prove the multiplicity result by using Theorem 2.2 for $\tilde{I}, C$, $\tilde{S_{23}}(\rho), \Delta_{12}\left(\tilde{R}, R_{1}\right)$ and $\Sigma_{12}\left(\tilde{R}, R_{1}\right)$. Now we have the following linking geometry for $\tilde{I}$.

Lemma 3.2. Assume that $G$ satisfies the conditions $(G 1)-(G 3)$, and that $j_{0}, j_{1}$ are negative integers with $j_{1}<j_{0}<0$. Then there exist a small number $\delta_{1}>0, R>\rho>0, R_{1}>0, R>1$ and $\rho>0$ with $R>\rho$ such that for any $\lambda$ with $j_{1}-\delta_{1}<\lambda<j_{1}<j_{0}<0$,

$$
\sup _{\tilde{z} \in \Sigma_{12}\left(\tilde{R}, R_{1}\right)} \tilde{I}(\tilde{z})<0<\inf _{\tilde{w} \in S_{23}(\rho)} \tilde{I}(\tilde{w})
$$

Moreover

$$
-\infty<\inf _{\tilde{w} \in B_{23}(\rho)} \tilde{I}(\tilde{w}), \quad \sup _{\tilde{z} \in \Delta_{12}\left(\tilde{R}, R_{1}\right)} \tilde{I}(\tilde{z})<\infty .
$$

Proof. It suffices to show that there exist $R>\rho>0, R_{1}>0$ and $R>1$ such that for $z=\psi(\tilde{z}), w=\psi(\tilde{w})$,

$$
\sup _{z \in \Sigma_{12}\left(R, R_{1}\right)} I(z)<\inf _{w \in S_{23}(\rho)} I(w) .
$$


because

$$
\sup _{\tilde{\varepsilon} \in \Sigma_{12}\left(\tilde{R}, R_{1}\right)} \tilde{I}(\tilde{z})=\sup _{z \in \Sigma_{12}\left(R, R_{1}\right)} I(z), \quad \inf _{\tilde{w} \in S_{23}(\rho)} \tilde{I}(\tilde{w})=\inf _{w \in S_{23}(\rho)} I(w) .
$$

Let $z=z_{1}+z_{2} \in X_{1} \oplus X_{2}$. By $(G 2)$ we have

$$
\begin{aligned}
I(z)= & \frac{1}{2}\left(\left\|\left|A_{\epsilon}\right|^{\frac{1}{2}} z_{+}\right\|_{L^{2}}^{2}+\left\|\left|A_{\epsilon}\right|^{\frac{1}{2}} M_{+} z_{0}\right\|^{2}-\left\|\left(-\left|A_{\epsilon}\right|\right)^{\frac{1}{2}} z_{-}\right\|_{L^{2}}^{2}\right. \\
& \left.-\left\|\left(-\left|A_{\epsilon}\right|\right)^{\frac{1}{2}} M_{-} z_{0}\right\|^{2}\right)-\int_{0}^{2 \pi}\left[\frac{1}{2}(\lambda+\epsilon) z^{2}+G(t, z(t))\right] d t \\
\leq & \frac{j_{1}+\epsilon-\lambda-\epsilon}{2}\left\|z_{2}\right\|_{L^{2}}^{2}+\frac{j_{0}+\epsilon-\lambda-\epsilon}{2}\left\|z_{2}\right\|_{L^{2}}^{2} \\
& -\int_{0}^{2 \pi}\left[\alpha_{1}|z|^{p_{1}+1}-\beta_{1}\right] d t . \\
\leq & \frac{\delta_{1}}{2}\left\|z_{2}\right\|_{L^{2}}^{2}+\frac{j_{0}+\epsilon-\lambda-\epsilon}{2}\left\|z_{2}\right\|_{L^{2}}^{2}-\int_{0}^{2 \pi}\left[\alpha_{1}|z|^{p_{1}+1}-\beta_{1}\right] d t .
\end{aligned}
$$

Since $j_{0}-\lambda>0$ and $p_{1}+1>2$, there exist a small number $\delta_{1}>0, R>0$, $R_{1}>0$ and $R>1$ such that for $z \in X_{1} \oplus X_{2}, \sup _{z \in \Sigma_{12}\left(R, R_{1}\right)} I(z)<0$. If $z \in \Delta_{12}\left(R, R_{1}\right)$, then $\sup _{z \in \Delta_{12}\left(R, R_{1}\right)} I(z)<\frac{\delta_{1}}{2}\left\|z_{2}\right\|_{L^{2}}^{2}+\frac{j_{0}+\epsilon-\lambda-\epsilon}{2}\left\|z_{2}\right\|_{L^{2}}^{2}+$ $2 \pi \beta_{1}<\infty$. On the other hand, if $z \in X_{2} \oplus X_{3}$, then

$$
\begin{aligned}
I(z)= & \frac{1}{2}\left(\left\|\left|A_{\epsilon}\right|^{\frac{1}{2}} z_{+}\right\|_{L^{2}}^{2}+\left\|\left|A_{\epsilon}\right|^{\frac{1}{2}} M_{+} z_{0}\right\|^{2}-\left\|\left(-\left|A_{\epsilon}\right|\right)^{\frac{1}{2}} z_{-}\right\|_{L^{2}}^{2}\right. \\
& \left.-\left\|\left(-\left|A_{\epsilon}\right|\right)^{\frac{1}{2}} M_{-} z_{0}\right\|^{2}\right)-\int_{0}^{2 \pi}\left[\frac{1}{2}(\lambda+\epsilon) z^{2}+G(t, z(t))\right] d t \\
& \geq \frac{j_{1}+\epsilon-\lambda-\epsilon}{2}\|z\|_{L^{2}}^{2}-\int_{0}^{2 \pi} \alpha_{2}|z|^{p_{2}+1} d t .
\end{aligned}
$$

Since $j_{1}-\lambda>0$ and $p_{2}+1>2$, there exists a small number $\rho>0$ with $R>\rho>0$ such that for $z \in X_{2} \oplus X_{3}$, inf $f_{z \in S_{23}(\rho)} I(z)>0$. If $z \in B_{23}(\rho)$, then $i n f_{z \in B_{23}(\rho)} I(z)>-\infty$. Thus we prove the lemma.

Lemma 3.3. Assume that $G$ satisfies the conditions $(G 1)-(G 3)$, and that $j_{0}, j_{1}$ are negative integers with $j_{1}<j_{0}<0$. Then for any $\lambda$ with $j_{1}-1 \leq \lambda<0, \tilde{I}$ has no critical point $\tilde{z}$ such that $\tilde{I}(\tilde{z})=c$ and $\tilde{z} \in \partial C$, where $\inf _{\tilde{z} \in B_{23}(\rho)} \tilde{I}(\tilde{z}) \leq c \leq \sup _{\tilde{z} \in \Sigma_{12}\left(\tilde{R}, R_{1}\right)} \tilde{I}(\tilde{z})<0$.

Proof. It suffices to prove that $I$ has no critical point $z=\psi(\tilde{z})$ such that $I(z)=c$ and $z \in X_{1} \oplus X_{3}$. We notice that from Lemma 3.2, for 
fixed $z_{1} \in X_{1}$, the functional $z_{3} \mapsto I\left(z_{1}+z_{3}\right)$ is weakly convex in $X_{3}$, while, for fixed $z_{3} \in X_{3}$, the functional $z_{1} \mapsto I\left(z_{1}+z_{3}\right)$ is strictly concave in $X_{1}$. Moreover $(0,0)$ is a critical point in $X_{1} \oplus X_{3}$ with $I(0,0)=0$. So if $z=z_{1}+z_{3}$ is another critical point for $\left.I\right|_{X_{1} \oplus X_{3}}$, then we have

$$
0=I(0,0) \leq I\left(z_{3}\right) \leq I\left(z_{1}+z_{3}\right) \leq I\left(z_{1}\right) \leq I(0,0)=0 .
$$

So $I\left(z_{1}+z_{3}\right)=I(0,0)=0$.

Lemma 3.4. Assume that $G$ satisfies the conditions $(G 1)-(G 3)$, and that $j_{0}, j_{1}$ are negative integers with $j_{1}<j_{0}<0$. Then there exists a constant $\delta_{2}>0$ such that for any $\lambda$ with $j_{1}-1-\delta_{2} \leq \lambda<j_{1}<$ $j_{0}<0$, $\tilde{I}$ has no critical point $\tilde{z}$ such that $\inf _{\tilde{z} \in B_{23}(\rho)} \tilde{I}(\tilde{z}) \leq \tilde{I}(\tilde{z}) \leq$ $\sup _{\tilde{z} \in \Sigma_{12}\left(\tilde{R}, R_{1}\right)} \tilde{I}(\tilde{z})<0$ and $\tilde{z} \in \partial C$.

Proof. It suffices to show that $I(z)$ has no critical point $z$ such that $\inf _{z \in B_{23}(\rho)} I(z) \leq I(z) \leq \sup _{z \in \Sigma_{12}\left(R, R_{1}\right)} I(z)<0$ and $z \in X_{1} \oplus X_{3}$. By contradiction we suppose that we can find two sequences $\left(\lambda_{n}\right)_{n}$ in $R$ with $\lambda_{n} \in\left(j_{1}-1-\delta_{2}, j_{1}\right)$ and $\left(z_{n}\right)_{n}$ in $X_{1} \oplus X_{3}$ such that $\lambda_{n} \rightarrow \lambda \in\left[j_{1}-1, j_{1}\right)$, $\inf _{z \in B_{23}(\rho)} I(z) \leq I(z) \leq \sup _{z \in \Sigma_{12}\left(R, R_{1}\right)} I(z)<0$ and $\left.D I\right|_{X_{1} \oplus X_{3}}\left(z_{n}\right)=0$. We claim that $\left(z_{n}\right)_{n}$ is bounded. If not we can suppose that $\left\|z_{n}\right\| \rightarrow$ $+\infty$ and set $w_{n}=\frac{z_{n}}{\left\|z_{n}\right\|}$. Since $w_{n}$ is bounded, up to a subsequence $w_{n}$ converges weakly to $w_{0}$, for some $w_{0} \in X_{1} \oplus X_{3}$. Furthermore since $P_{X_{1}} z_{n} \in E_{-},\left\|P_{+} P_{X_{1}} z_{n}\right\|=0$ and we have

$$
\begin{aligned}
\left\langle D I\left(z_{n}\right), P_{X_{1}} z_{n}\right\rangle= & \left\|P_{+} P_{X_{1}} z_{n}\right\|^{2}-\left\|P_{-} P_{X_{1}} z_{n}\right\|^{2} \\
& -\left\langle(\lambda+\epsilon) z_{n}+G_{z}\left(t, z_{n}\right), P_{X_{1}} z_{n}\right\rangle \\
= & -\left\|P_{-} P_{X_{1}} z_{n}\right\|^{2}-\left\langle(\lambda+\epsilon) z_{n}+G_{z}\left(t, z_{n}\right), P_{X_{1}} z_{n}\right\rangle \\
\longrightarrow & 0 .
\end{aligned}
$$

Moreover since $P_{X_{3}} z_{n} \in E_{+},\left\|P_{-} P_{X_{3}} z_{n}\right\|=0$ and we have

$$
\left\langle D I\left(z_{n}\right), P_{X_{3}} z_{n}\right\rangle=\left\|P_{+} P_{X_{3}} z_{n}\right\|^{2}-\left\langle(\lambda+\epsilon) z_{n}+G_{z}\left(t, z_{n}\right), P_{X_{3}} z_{n}\right\rangle \longrightarrow 0 .
$$

Adding (3.9) to (3.10), we have

$\lim _{n \rightarrow \infty}\left(\left\|P_{+} P_{X_{3}} z_{n}\right\|^{2}-\left\|P_{-} P_{X_{1}} z_{n}\right\|^{2}\right)=\lim _{n \rightarrow \infty}\left\langle(\lambda+\epsilon) z_{n}+G_{z}\left(t, z_{n}\right), P_{X_{1} \oplus X_{3}} z_{n}\right\rangle$.

Dividing (3.11) by $\left\|z_{n}\right\|^{2}$ and going to the limit, we get

$$
\left\|P_{+} P_{X_{3}} w_{0}\right\|^{2}-\left\|P_{-} P_{X_{1}} w_{0}\right\|^{2}=\left\langle(\lambda+\epsilon) w_{0}, P_{X_{1} \oplus X_{3}} w_{0}\right\rangle,
$$


from which $w_{0}$ is the unique solution of the linear equation

$$
A_{\epsilon} z=(\lambda+\epsilon) z .
$$

Since $\lambda \notin \sigma(A), w_{0}=0$, which is a contradiction to the fact $\left\|w_{0}\right\|=1$. Thus $\left(z_{n}\right)_{n}$ is bounded. By the same arguments used for $\left(w_{n}\right)_{n}$, we get, up to a subsequence, $\left(z_{n}\right)_{n}$ converges strongly to a point $z \in X_{1} \oplus X_{3}$ with $\inf _{z \in B_{23}(\rho)} I(z) \leq I(z) \leq \sup _{z \in \Sigma_{12}\left(R, R_{1}\right)} I(z)<0$ and $\left.D I\right|_{X_{1} \oplus X_{3}}(z)=0$, which contradicts Lemma 3.3. Thus we prove the lemma.

Lemma 3.5. The functional $-\tilde{I}$ satisfies the $(P . S .)_{-\tilde{c}}^{*}$ condition with respect to $\left(C_{n}\right)_{n}$ for any $-\tilde{c}$ such that

$$
0<\inf _{\tilde{z} \in \Sigma_{12}\left(\tilde{R}, R_{1}\right)}(-\tilde{I})(\tilde{z}) \leq-\tilde{c} \leq \sup _{\tilde{z} \in B_{23}(\rho)}(-\tilde{I})(\tilde{z}) .
$$

Proof. Let $\left(h_{n}\right)_{n}$ be a sequence in $N$ with $h_{n} \rightarrow+\infty$ and $\left(\tilde{z_{n}}\right)_{n}$ be a sequence in $C$ with $\tilde{z_{n}} \in C_{h_{n}}$ for all $n,-\tilde{I}\left(\tilde{z_{n}}\right) \rightarrow-\tilde{c}$ and $\operatorname{grad}_{C_{h_{n}}}^{-}\left(-\left.\tilde{I}\right|_{E_{h_{n}}}\right)\left(\tilde{z_{n}}\right)$ $\rightarrow 0$. Set $z_{n}=\Psi\left(\tilde{z_{n}}\right)$. Then $I\left(z_{n}\right) \rightarrow c$. We first consider the case $\tilde{z_{n}} \notin \partial C_{h_{n}}$ for large $n$. Since for large $n P_{E_{n}} \circ P_{X_{2}}=P_{X_{2}} \circ P_{E_{n}}=P_{X_{2}}$, we have

$$
\begin{aligned}
& \operatorname{grad}_{C_{h_{n}}}^{-}(-\tilde{I})\left(\tilde{z_{n}}\right)=P_{E_{h_{n}}} \Psi^{\prime}\left(\tilde{z_{n}}\right) D(-I)\left(z_{n}\right)=\Psi^{\prime}\left(\tilde{z}_{n}\right)\left(P_{E_{h_{n}}} D(-I)\left(z_{n}\right)\right) \\
& =P_{E_{h_{n}}} P_{X_{1} \oplus X_{3}} D(-I)\left(z_{n}\right)+P_{E_{h_{n}}}\left(1-\frac{1}{\left\|P_{X_{2}} \tilde{z_{n}}\right\|_{E}}\right) P_{X_{2}} D(-I)\left(z_{n}\right) \rightarrow 0 .
\end{aligned}
$$

Thus

$$
\begin{aligned}
& P_{X_{1} \oplus X_{3}} P_{E_{h_{n}}} D(-I)\left(z_{n}\right) \rightarrow 0 \quad \text { and } \\
& \left(1-\frac{1}{\left\|P_{X_{2}} \tilde{z_{n}}\right\|_{E}}\right) P_{X_{2}} D(-I)\left(z_{n}\right) \rightarrow 0 .
\end{aligned}
$$

It is impossible that $\left\|P_{X_{2}} \tilde{z_{n}}\right\|_{E} \rightarrow 1$ because $\operatorname{dist}\left(z_{n}, X_{2}\right) \rightarrow 0$. Thus $P_{E_{h_{n}}} D(-I)\left(z_{n}\right) \rightarrow 0$. Using (P.S. $)_{c}^{*}$ for $I$ of Lemma 3.1 it follows that $\left(z_{n}\right)_{n}$ has a subsequence $\left(z_{k_{n}}\right)_{n}$ such that $z_{k_{n}} \rightarrow z$ for some $z$ in $X_{2}$. Since $\Psi$ is invertible in $\operatorname{int}(C), z_{\tilde{k}_{n}} \rightarrow \Psi^{-1}(z)$. Next we consider the case $\tilde{z_{n}} \in \partial C_{h_{n}}$ for infinitely many $n$. We claim that this case cannot occur. If $\tilde{z_{n}} \in \partial C_{h_{n}}$, then $\left\|P_{X_{2}} \tilde{z_{n}}\right\|_{E}=1$. Thus we have

$$
\begin{aligned}
\operatorname{grad}_{C_{h_{n}}}^{-}(-\tilde{I})\left(\tilde{z_{n}}\right)=P_{E_{h_{n}}} & \left(P_{X_{1} \oplus X_{3}} D(-I)\left(z_{n}\right)\right. \\
& \left.-\left\langle D(-I)\left(z_{n}\right), P_{X_{2}} \tilde{z_{n}}\right\rangle^{+} P_{X_{2}} \tilde{z_{n}}\right) \rightarrow 0 .
\end{aligned}
$$

Using the properties of the projections, we get

$$
P_{E_{h_{n}}} P_{X_{1} \oplus X_{3}} D(-I)\left(z_{n}\right) \rightarrow 0,
$$


which contradicts to Lemma 3.3. In fact, let $\tilde{z}$ be the limit point of the subsequence $\tilde{z}_{k_{n}}$ of $\tilde{z_{n}}$, then $\tilde{z} \in \partial C$ and

$$
\operatorname{grad}_{C}^{-}(-\tilde{I})(\tilde{z})=P_{X_{1} \oplus X_{3}} \operatorname{grad}(-I)(z)-\left\langle\operatorname{grad}(-I)(z), P_{X_{2}} \tilde{z}\right\rangle P_{X_{2}} \tilde{z} .
$$

\section{Proof of TheOREM 1.1}

Assume that $G$ satisfies the conditions $(G 1)-(G 3)$, and that $j_{0}, j_{1}$ are negative integers with $j_{1}<j_{0}<0$. Let $\lambda \notin Z$ and $\lambda<0$. We note that the critical points of the functional $\tilde{I}$ coincide with the critical points of the functional $-\tilde{I}$. Thus it suffices to find the number of the critical points of $-\tilde{I}$, which is appropriate functional for applying Theorem 2.2, to find the number of the critical points of $I$. The common part of $\left(j_{1}-\delta_{1}, j_{1}\right)$ and $\left(j_{1}-1-\delta_{2}, j_{1}\right)$ is

$$
\left(j_{1}-\delta_{1}, j_{1}\right)
$$

where $\delta_{1}$ and $\delta_{2}$ are small numbers introduced in Lemma 3.2 and Lemma 3.4 respectively. Let us set

$$
\delta=\delta_{1}
$$

By Lemma 3.2, there exist $R>\rho>0, R_{1}>0, R>1$ and $\rho>0$ with $R>\rho$ such that for any $\lambda$ with $j_{1}-\delta<\lambda<j_{1}<j_{0}<0$,

$$
\begin{aligned}
\sup _{\tilde{z} \in S_{23}(\rho)}(-\tilde{I})(\tilde{z})=\sup _{z \in S_{23}(\rho)}(-I)(z)<0 & <\inf _{z \in \Sigma_{12}\left(R, R_{1}\right)}(-I)(z) \\
& =\inf _{\tilde{z} \in \Sigma_{12}\left(\tilde{R}, R_{1}\right)}(-\tilde{I})(z) .
\end{aligned}
$$

and

$$
\begin{gathered}
\inf _{\tilde{z} \in \Delta_{12}\left(\tilde{R}, R_{1}\right)}(-\tilde{I})(z)=-\sup _{\tilde{z} \in \Delta_{12}\left(\tilde{R}, R_{1}\right)} \tilde{I}(z)>-\infty, \\
\sup _{\tilde{z} \in B_{23}(\rho)}(-\tilde{I})(\tilde{z})=-\inf _{\tilde{z} \in B_{23}(\rho)} \tilde{I}(\tilde{z})<\infty,
\end{gathered}
$$

so the condition (a) of Theorem 2.2 for the functional $-\tilde{I}$ is satisfied. By Lemma 3.5, the functional $-\tilde{I}$ satisfies the $(P . S .)_{-\tilde{c}}^{*}$ condition with respect to $\left(C_{n}\right)_{n}$ for any $-\tilde{c} \in[\alpha, \beta]$, where $\alpha=\inf _{\tilde{z} \in \Sigma_{12}\left(\tilde{R}, R_{1}\right)}(-\tilde{I})(\tilde{z})$ and $\beta=\sup _{\tilde{z} \in B_{23}(\rho)}(-\tilde{I})(\tilde{z})$, so the condition (b) of Theorem 2.2 is satisfied. By Lemma 3.4, for any $\lambda$ with $j_{1}-\delta<\lambda<j_{1}<j_{0}<0, \tilde{I}$ has no critical point $\tilde{z}$ such that $\inf _{\tilde{z} \in B_{23}(\rho)} \tilde{I}(\tilde{z}) \leq \tilde{I}(\tilde{z}) \leq \sup _{\tilde{z} \in \Sigma_{12}\left(\tilde{R}, R_{1}\right)} \tilde{I}(\tilde{z})<0$ and $\tilde{z} \in \partial C$, so the condition (c) of Theorem 2.2 is satisfied. Thus by 
Theorem 2.2, for any $\lambda$ with $j_{1}-\delta<\lambda<j_{1}<j_{0}<0$ there exists two lower critical points $\tilde{z_{1}}, \tilde{z_{2}}$ for $-\tilde{I}$ on $C$ such that

$$
0<\inf _{\tilde{z} \in \Sigma_{12}\left(\tilde{R}, R_{1}\right)}(-\tilde{I})(\tilde{z}) \leq(-\tilde{I})\left(\tilde{z}_{i}\right) \leq \sup _{\tilde{z} \in B_{23}(\rho)}(-\tilde{I})(\tilde{z}), \quad i=1.2
$$

Thus the functional $I$ has at least two lower critical points $z_{1}, z_{2}$ on $X_{2}$ with

$$
\inf _{z \in B_{23}(\rho)} I(z) \leq I\left(z_{i}\right) \leq \sup _{z \in \Sigma_{12}\left(R, R_{1}\right)} I(z)<0, \quad i=1.2
$$

Thus system (1.2) has at least two nontrivial solutions. Thus Theorem 1.1 is proved.

\section{References}

[1] K. C. Chang, Infinite dimensional Morse theory and multiple solution problems, Birkhäuser, (1993).

[2] M. Degiovanni, Homotopical properties of a class of nonsmooth functions, Ann. Mat. Pura Appl. 156 (1990), 37-71.

[3] M. Degiovanni, A. Marino, and M. Tosques, Evolution equation with lack of convexity, Nonlinear Anal. 9 (1985), 1401-1433.

[4] G. Fournier, D. Lupo, M. Ramos, and M. Willem, Limit relative category and critical point theory, Dynam. Report, 3 (1993), 1-23.

[5] T. Jung and Q. H. Choi, On the number of the periodic solutions of the nonlinear Hamiltonian system, Nonlinear Analysis TMA, Vol. 71, No. 12 e1100-e1108 (2009).

[6] T. Jung and Q. H. Choi, Periodic solutions for the nonlinear Hamiltonian systems, Korean J. Math. 17 (3) (2009), 331-340.

[7] T. Jung and Q. H. Choi, Existence of four solutions of the nonlinear Hamiltonian system with nonlinearity crossing two eigenvalues, Boundary Value Problems, Volume 2008, 1-17.

[8] A. M. Micheletti and A. Pistoia, On the number of solutions for a class of fourth order elliptic problems, Communications on Applied Nonlinear Analysis 6 (2) (1999), 49-69.

[9] P. H. Rabinowitz, Minimax methods in critical point theory with applications to differential equations, CBMS. Regional Conf. Ser. Math., 65, Amer. Math. Soc., Providence, Rhode Island (1986). 
Tacksun Jung

Department of Mathematics

Kunsan National University

Kunsan 573-701,Korea

E-mail: tsjung@kunsan.ac.kr

Q-Heung Choi

Department of Mathematics Education

Inha University

Incheon 402-751, Korea

E-mail: qheung@inha.ac.kr 